\title{
Gestión Estratégica como Plataforma Impulsora del Capital Social en las Pymes
}

\section{Strategic Management as an Impulse Platform for Social Capital in SMEs}

\author{
Walter Bermudez \\ walterbeme@hotmail.es \\ Universidad del Atlántico \\ Colombia \\ https://orcid.org/0000-0003-4380-4247 \\ Luis Urueta \\ luismariano71119@gmail.com \\ Universidad del Atlántico \\ Colombia \\ https://orcid.org/0000-0003-22782448
}

\begin{abstract}
RESUMEN
El propósito general de este estudio analizar la gestión estratégica como plataforma impulsora del capital social en las pymes. La investigación se enmarca con un enfoque pospositivista de corte cualitativa, bajo un paradigma fenomenológico hermenéutico, se basa en la recopilación de los fundamentos de diversos autores tales como Portes (2009), Francés (2008), George y Jones (2010). La información fue recabada mediante las técnicas de observación directa y entrevistas cualitativas. A efectos de la muestra se tomaron (4) sujetos. De manera conclusiva se manifiesta que las Pymes son conscientes de que la inversión en el capital social les genera un alto valor agregado, de la mano con el trabajo social y el fortalecimiento de la formación en su talento humano, requiriendo orientar sus esfuerzos hacia la concepción, desarrollo e evaluación de metas empresariales compatibles con los tres vértices del desarrollo sostenible que son el valor social, económico y medioambiental.
\end{abstract}

Descriptores: Gestión Estratégica; Plataforma Impulsora; Capital Social; Pymes. 


\section{ABSTRACT}

The general purpose of this study is to analyze strategic management as a driving platform for social capital in SMEs. The research is framed with a qualitative postpositivist approach, under a hermeneutical phenomenological paradigm, based on the compilation of the foundations of various authors such as Portes (2009), French (2008), George and Jones (2010). The information was collected through direct observation techniques and qualitative interviews. For the purposes of the sample, (4) subjects were taken. In a conclusive way it is stated that SMEs are aware that investment in social capital generates a high added value, hand in hand with social work and the strengthening of training in their human talent, requiring to direct their efforts towards conception, development and evaluation of business goals compatible with the three vertices of sustainable development that are social, economic and environmental value.

Descriptors: Strategic Management; Impulsora Platform; Social Capital; SMEs.

Recibido: 15 de marzo del 2019

Aprobado: 1 de mayo del 2019

\section{INTRODUCCIÓN}

El contexto de las PYMES está cambiando vertiginosamente esto causa efectos colaterales en su gestión, viéndose exigidas a adecuarse a nuevas formas de realizar el trabajo y adaptarse a las características sociales del entorno, para enfrentar las eventuales amenazas que este les ofrece. Debido a lo anterior, se puede presumir la falta de estrategias las cuales hagan sustentable el desarrollo de las PYMES, coherente con la misión, visión y principios estratégicos planteados en su nacimiento como empresa.

Por ende, se puede creer que las PYMES son una organización social, donde cada miembro interactúa constantemente con los demás, pudiéndose generar mayor cohesión y conexión entre sus miembros, esto logra un mayor desarrollo, aunado con la cultura y el ambiente, esto se convierte en un reforzador importante para el impulso de acciones de cara a alcanzar propósitos.

Es una realidad que, las pequeñas y medianas empresas, PYMES, se encuentran sumergidas en diversos procesos de trasformación socioculturales, cambios en el mercado y complejidad que obedecen a la globalización, a las tecnologías, la 
diversidad social y las políticas económicas, las cuales emergen como repuestas a estas situaciones, al mismo tiempo, a esta realidad se pueden sumar, la inestabilidad económica y los diferentes factores de seguridad que impactan en estas. En tal sentido, las pequeñas y medianas empresas (PYMES) desarrollan sus acciones en un ambiente de constante cambio, con una fuerte injerencia de la naturaleza social del contexto, obligando desde esta perspectiva a aprovechar al máximo el componente social para adoptar una posición estratégica.

Por consiguiente, las PYMES necesitan aprovechar para su direccionamiento estratégico variables que se ajusten más a la estructura social, por cuanto con ello se alcanza un desarrollo empresarial, así como el desarrollo económico, interviniendo la cultura, subcultura y los grupos sociales a los que pertenece o se relaciona un individuo como factor determinante de su comportamiento económico, pudiendo ser concluyente para este propósito.

Ante este escenario, se valdría considerar este capital la base organizacional que promueve la gestión estratégica como un estilo de dirección acorde para manejar adecuadamente los recursos, la tecnología y el talento humano, en miras de lograr la eficiencia así como la eficacia, es decir, las PYMES podrían responder con eficiencia a las exigencia del entorno, asimismo con eficacia a la producción de productos que permitan satisfacer necesidades en los mercados de los cuales se esté inmerso.

En tal sentido, una gestión estratégica fundamentada en el capital social lograría que las PYMES alcanzarán mayor nivel de competitividad en el mercado, a la vez más sostenibilidad, donde la colectividad del talento humano facilita tal resultado cuando se presenta una plataforma social compacta y altamente motivada, de lo contrario, lo más probable es que se presenten serias complicaciones para responder a las necesidades del mercado así como a las expectativas de los accionistas, afectando notoriamente la finanzas de la empresa, además de los aspectos sociales de unidad.

Tomando en consideración lo planteado con anterioridad se podría decir que la investigación plantea una gestión estratégica más humana que formule acciones encaminada a reforzar vínculos de unión, para concebir un ambiente adecuado de trabajo y fortalecer al capital social, generando un impacto positivo en la ejecución 
de las estrategias.

\section{DESARROLLO TEÓRICO}

En la búsqueda de sustento teórico que aporte conocimientos, se presenta el sistema referencial desde la postura de distintos autores, los cuales a través de la identificación, descripción, comprensión y análisis plasman su perspectiva en relación con la categoría y subcategorías que le dan forma al estudio:

\section{Gestión Estratégica como plataforma impulsora del capital social}

Actualmente, existen grandes avances en todas las áreas del quehacer humano, logrando alcanzar un alto nivel de desarrollo tanto en la ciencia como en la tecnología. Un siglo atrás parecería una fantasía pensar que los seres humanos pudiesen estar interconectados de un continente a otro, a través de sistemas de comunicación como el internet, celular, otros, estos junto al transporte moderno han facilitado el comercio entre diversos países.

Desde esta concepción, la gerencia de organizaciones modernas, con el fin de permitir seguir siendo un actor clave en el desarrollo económico-social de la población, hace frente a nuevos retos relevantes para lograr los niveles de competitividad, sostenibilidad y sustentabilidad necesarios para el alcance de un equilibrio sano, por lo que promueve la gestión estratégica como plataforma impulsora del capital social.

En este sentido, para George y Jones (2010, p.231), la gestión estratégica "es un proceso que consiste en la identificación de metas y líneas de acción apropiadas a objeto de establecer que acciones emprender para lograr los objetivos". En correspondencia con estos planteamientos, según Cortina y Martínez (2008), la gestión estratégica, intenta construirse racionalmente, y así pueda reflexionar sobre la acción del hombre, hasta el punto crucial donde este pueda decidir entre varias opciones estratégicas como lo sostienen Thompson, Strickland y Gamble (2010), consiste en asegurarse de que los objetivos, tácticas, así como las estrategias emprendidas pasen la prueba del escrutinio moral.

En el mismo orden, el capital social se ha convertido en un paradigma que llama la atención de las grandes entidades mundiales; convirtiéndose en una 
herramienta primordial para el desarrollo de la sociedad por medio de la integración de valores morales a las posibilidades de su entorno. Por esta razón, es necesario concertar la satisfacción de los beneficios particulares en función del interés social bajo los parámetros de justicia social, es decir, criterios ético económicos. Dentro de la visión del desarrollo económico de una nación, además de los grandes representantes (Estado, mercado y la sociedad) se necesita la cooperación de los individuos, gobierno, familias, instituciones, empresas, con sus respectivas creencias y valores, principios, comportamientos.

Considerando esto, Portes (2009, p.34), explica que se entiende por capital social "aquel activo intangible que se manifiesta en la capacidad de confianza, valores cívicos y asociatividad que pueda lograr la sociedad". Este término es utilizado como una mezcla de distintas disciplinas estableciendo un marco conceptual conjunto. A pesar de que la economía convencional lo ha desconocido, el capital social tiene un peso significativo en las posibilidades de desarrollo económico de las sociedades,

Por otra parte, los niveles de desarrollo que han logrado el comercio, la industria y el sector de servicios le ha permitido a la humanidad elevar se nivel de vida. Al respecto, según Francés (2008), el capital social de la empresa se ha convertido en protagonista, responsable de gran parte del auge económico, al concebir productos o servicios que han permitido mejorar la vida de la sociedad.

Dentro del mismo orden de ideas, al incorporar la gestión estratégica dentro del capital social, con la participación de los accionistas, en primera instancia la alta gerencia, para luego sensibilizar a la gerencia media, operativa, así como a todos los miembros, para desarrollar estrategias empresariales eficientes que generen como resultado una exitosa participación de mercado, rentabilidad, calidad integrada (procesos, productos y personas).

Lo anterior, trae como consecuencia un valor agregado para la organización, lo que a su vez promueve el desarrollo de activos intangibles, los cuales según Villafañe (2007) son aquellos activos de una empresa que no poseen materialidad, como la reputación corporativa, del cual se desprenden otros intangibles como: el capital relacional, capital intelectual, cultura organizacional, identidad corporativa, entre otros. 
Estos activos intangibles hacen, para Olcese, Rodríguez y Alfaro (2008), que se convierta en uno de los propósitos más influyentes en la gestión estratégica, y se denomina "la gestión de los intangibles", es decir, la gestión integral de los activos intangibles, los cuales aportan el mayor valor a las empresas, la cual se basa principios macro a tener en consideración por los estrategas en su planificación. Es decir, integrar la misión, visión, estrategias, políticas, objetivos, tácticas entre otros elementos de la gestión estratégica, permitiendo el logro de los objetivos organizacionales, con un alto grado de compromiso con los valores morales, satisfaciendo las necesidades de clientes, empleados, proveedores, accionistas entre otros stakeholdres.

Según George y Jones (2010) la gestión consiste en el proceso de seleccionar e identificar tanto las metas como las líneas de acción adecuadas, para el logro de metas de cualquier organización, considerándola como una de las funciones principales de la gerencia. En este sentido, si todos dentro de la empresa se enfocan en el esfuerzo de ser efectivos, las personas deben saber que se espera de ellos, allí se emplea la función de planeación, la más esencial de todas las funciones gerenciales.

Al considerar el campo empresarial ocupado por esta investigación, la gestión estratégica es el modelo o plan que integra las principales metas, políticas y cadenas de acciones de una organización dentro de una totalidad. Por último, según George y Jones (2010), la gestión estratégica consiste en un grupo o conjunto de decisiones y acciones relacionadas entre sí para el logro de las metas de una organización, de allí, la estrategia como la planeación sean tanto un proceso de toma de decisiones para formular o seleccionar las mejores alternativas de acción.

En este orden de ideas, cabe afirmar que el capital social adquiere relevancia, por cuanto le toca decidir de manera asertiva y éticamente las acciones a seguir para lograr los objetivos deseados por la directiva de la organización. En consecuencia, al elegir una alternativa estratégica, se encuentra de nuevo el elemento decisional como factor clave para el éxito corporativo.

Por ello, los investigadores consideran que la empresa debe contar con un capital social que le permita conducirse, competir, en una combinación particular de 
planteamientos o escenarios competitivos y operativos para llevar a la organización en la dirección deseada, fortalecer su posición en el mercado, así como su competitividad y mejorar su desempeño.

\section{Gestión estratégica}

Cuando un gerente pasa de los niveles tácticos-funcionales (relaciones industriales, finanzas, mercadeo, producción, entre otros) a los estratégicos, es común que tenga poco conocimiento y por ende no domina las principales teorías, las técnicas y las herramientas adecuadas para la administración total de la empresa, es por ello, que debe aumentar sus conocimientos de la administración general, personal, financiera o comercial a otra forma de gestión: la estratégica de la organización.

En este sentido, Sallenave (2010, p.101) la gerencia estratégica concerniente a la empresa moderna "requiere de conocimientos técnicos, así como de la comprensión de los factores sociales, estructurales y políticos que le dan forma". La complejidad es cada vez más exigente, con el paso del tiempo y con la cantidad de situaciones que se presentan.

Es normal que la nueva visión se presente con gran incertidumbre, debido a la falta de experiencia no sabe por dónde comenzar, al enfrentarse a nuevas situaciones empresariales, en donde se entrelazan problemas técnicos y humanos. En consecuencia, se debe adquirir conocimientos especializados con otros conceptos, métodos e ideas en el proceso de análisis de problema para la adopción de decisiones, como lo es la conducción estratégica de la organización. De acuerdo con, Strickland (2009, p.78), la estrategia de una empresa se puede definir como "el plan de acción que tiene la administración para posicionar a la compañía en la arena de su mercado, competir con éxito, satisfacer a los clientes y lograr un buen desempeño del negocio". Tomando en cuenta esta definición, la estrategia entonces representa una amplia gama de acciones competitivas, así como una nueva misión organizacional que deben implantar los gerentes, para alcanzar el éxito deseado. Sin lugar a dudas, este enfoque estratégico moderno requiere de procesos profundos para adoptar decisiones, por cuanto se debe seleccionar el destino por medio de caminos seguros. 
Según Robinns (2008), la tarea se hace más compleja, al identificar y seleccionar un curso estratégico entre todas las opciones presentes, situación complicada, pues se debe justificar porque entre todas las trayectorias elegibles, se decidió seguir la dirección escogida confiando en que se configura como la mejor opción para posicionar a la compañía. En consecuencia, la selección e implantación de un proceso de gerencia estratégica conlleva a decisiones gerenciales entre gran cantidad de opciones e indica el compromiso empresarial y estilo de actuación. Los expertos en planificación y gerencia estratégica Robinns (2008), Chiavenato (2009) y Strickland (2009), coinciden que este proceso exista, debe estar representado por cinco actividades gerenciales indispensables:

1a) Formular una visión estratégica de la estructura de la organización, hacia donde debe dirigirse, con la finalidad de identificar los mejores escenarios y proporcionar un camino a largo plazo, identificar en qué clase de empresa quiere transformarse, estimulando internamente una visión compartida, con el objetivo de reafirmar el verdadero propósito estratégico.

$2^{a}$ ) Establecimiento de objetivos, es decir, la conversión de la visión estratégica en resultados muy claros de la práctica que quiere conseguir la organización. Esto es convertir los fundamentos gerenciales de la visión estratégica y de la misión de la empresa en indicadores de desempeño, instrumentos que pueden ser utilizados para medir el progreso, en otras palabras, los objetivos son los criterios guías para dar seguimiento al ejercicio y al progreso de una organización.

$3^{\text {a) }}$ Crear una estrategia, que garantice la consecución de los resultados deseados, esta pasa a ser la respuesta de la gerencia a los componentes tan fundamentales como si se debe estar concentrada en una o varias unidades estratégicas de negocio, es decir, son las acciones y enfoques de negocios que emplea la gerencia para garantizar un desempeño organizacional satisfactorio. Sin lugar a dudas la formulación e implantación de una estrategia exitosa debe ser una responsabilidad gerencial prioritaria en toda organización.

$4^{a}$ ) Implantar y ejecutar la estrategia seleccionada de una manera efectiva y eficiente, esto requiere de una valorización de los recursos tanto humanos como materiales para que la estrategia produzca los resultados esperados y así garantizar el momento oportuno al estilo de actuación seleccionado; así pues, 
esta actividad requiere de una competencia gerencial para innovar lo necesario para la implantación de la estrategia, ejecutarla en forma eficiente, garantizando así los resultados esperados.

$5^{\text {a }}$ ) Evaluar el desempeño y tomar las medidas correctivas tanto en la visión como en la misión, al igual que en el recorrido a largo plazo, los objetivos, la implantación de la estrategia, considerando los resultados reales obtenidos, las condiciones cambiantes del entorno, los procesos creativos y las nuevas oportunidades que se presenten. Este paso es necesario por cuanto la visión, los objetivos, la estrategia y el enfoque de la empresa a la implantación nunca son concluyentes.

\section{La base del capital social}

Desde la perspectiva de que el capital social tiene importantes implicaciones para el desempeño de las organizaciones, así como para los mismos ciudadanos, se puede decir entonces, este es un bien público basado en los aspectos de organización social, entre estos la confianza, las redes y reglas que superan los dilemas de la acción colectiva para alcanzar la eficiencia social.

El capital social es definido por Putnam (2007, p. 210) como los elementos de la organización social, tales como la confianza, las normas y las redes que establecen relaciones de reciprocidad activadas por una confianza social emergente de dos fuentes, las normas de reciprocidad y las "redes de compromiso ciudadano". Más ampliamente, el citado autor define como capital social los "rasgos de la organización social como confianza, normas y redes que pueden mejorar la eficiencia de la sociedad facilitando acciones coordenadas", la cual resulta de un "proceso histórico, cuyas tradiciones asociativas son preservadas mediante el capital social" y que como forma de organización "permite evitar los dilemas de la acción colectiva mediante lazos de confianza social".

En este sentido, las relaciones de confianza llegan a generar una confianza social o confianza generalizada, esto cuando prevalecen normas de reciprocidad y redes de compromiso cívico. Para Lechner, (2010), el capital social se expresa en la relación existente entre relaciones de confianza y cooperación cívica. Bajo esta 
visión, a partir de los hechos sociales y con la orientación de la economía, Putnam (2007, p. 212), define el capital social como "las características de organización social, tales como confianza, las normas y redes que pueden mejorar la eficiencia de la sociedad mediante la facilitación de las acciones coordinadas".

En concordancia, Etkin (2014), conceptualiza el capital social como los "mecanismos de la organización social tales como las redes, normas, y la confianza social que facilita la coordinación y cooperación para beneficios mutuos". Desde este punto de vista, el capital social permite una mayor comprensión sobre los mecanismos de los ambientes de competencia en todos mercados, lo que enfoca su análisis del capital social en el fenómeno del liderazgo.

El capital social pasa a ser un recurso acumulable creciente en la construcción social para la operacionalición de relaciones sociales. Es por lo tanto, un atributo colectivo más que una agregación de las conexiones sociales de los individuos, se puede explicar cómo el subproducto de otras actividades organizacionales y por lo tanto concierta un componente indispensable para la acción colectiva.

La acción colectiva debe ser estimulada por las instituciones propulsoras de actividades y relaciones de las personas. El capital social es un activo cuya propiedad conjunta entre los miembros y la organización beneficia a todos. Una organización nueva tiene la ventaja de poder crear su capital social organizacional, en tal forma que conserve un equilibrio óptimo entre los intereses individuales e intereses organizacionales.

El desarrollo social en un sentido sustentable depende en gran parte de la forma de capital social inherente a esa sociedad. Una comunidad cuenta con capital social cuando sus organizaciones se determinan por las relaciones de confianza que desarrollan, que hace predecible su comportamiento. La aceptación de la pluralidad política y cultural en procesos de autogestión y solidaridad facilita las bases morales, políticas e organizativas del desarrollo de las comunidades.

Los procesos que se dan en función de la sostenibilidad, pueden dar cierta estabilidad a los campos organizacionales, aunque estos siempre están evolucionando, resolviendo mediante consenso negociado socialmente las diferencias de interpretación. La capacidad de una comunidad se refleja en su 
nivel de desarrollo endógeno. Las organizaciones siguen estrategias habituales y las que siguen estrategias de nichos ocupan diferentes recursos ambientales, para dar respuestas innovadoras se forman vínculos interdependientes a otras organizaciones especializadas en otras industrias tales como estructuras de apoyo comunitario.

\section{PYMES como ente de acción del capital social}

Las pequeñas y medianas empresas, conocidas como PYMES, pueden ser descritas como una organización con características propias, que presentan dimensiones con ciertos límites ocupacionales y financieros preestablecidos por los estados o regiones. Las PYMES pasan hacer agentes con lógicas, intereses, culturas y un espíritu emprendedor específicos.

Desde la visión de Cleri (2013, P.25), la pequeña empresa "es una entidad independiente, creada para ser rentable, que no predomina en la industria a la que pertenece". Además, su venta anual en valores no excede un determinado tope, el número de individuos que la conforma no excede un determinado límite, como toda empresa, tiene aspiraciones, realizaciones, bienes materiales, capacidades técnicas y financieras, todo lo cual, le permite dedicarse a la producción, transformación y/o prestación de servicios para satisfacer determinadas necesidades existentes de la sociedad.

En este sentido, las PYMES sirven de tejido auxiliar a las grandes empresas, por lo que la mayor parte de las grandes empresas se valen de la subcontratación de empresas menores para realizar servicios u operaciones que de estar incluidas en el tejido de la gran corporación redundaría en un aumento de coste, de esta manera, existen actividades productivas donde es más apropiado trabajar con empresas pequeñas.

Explica Corma (2012), la mayor ventaja presente es las PYMES es su capacidad de cambiar velozmente su estructura productiva en el caso de variar las necesidades, esto es mucho más difícil en una gran empresa, con muchos empleados y gran capital invertido. Sin embargo, el acceso a mercados específicos aumenta su riesgo de quiebra, por lo que es importante para las PYMES ampliar su mercado o sus clientes. 
Lo anterior permite dilucidar, que las empresas pequeñas tienen más dificultad de encontrar financiación a un coste y plazo adecuados debido a su mayor riesgo. Para solucionar esto, Sandis y Ribeiro (2009), proponen para estas, recurrir a otras empresas con mayor capital, tomando en cuenta la rigidez laboral que presentan. Para la constitución y funcionamiento de las PYMES, es importante la formación previa del empleado, así como el uso tecnológico adecuado, ya que debido al pequeño volumen de beneficios presentado por estas empresas, no pueden dedicar fondos a la investigación, por eso deben asociarse con otras empresas o universidades.

\section{ESCENARIO METODOLÓGICO}

Desde esta perspectiva, la metodología implementada para el desarrollo de este estudio, considera en sus argumentos los diferentes factores los cuales involucran al entorno social y junto con este a los individuos, quien en definitiva son los determinantes de las técnicas que se utilizan para el análisis de los datos obtenidos y los procedimientos. De esta manera, se puede contextualizar el conocimiento de forma explícita, sin embargo, es importante considerar la epistemología, en atención a ello, es prominente dar respuesta a las interrogantes de manera que logre obtener hallazgos relevantes.

En el caso que ocupa a esta investigación, la misma, concibe la creación o generación de conocimiento desde el análisis directo de la realidad, el cual es, analizar la gestión estratégica como plataforma impulsora del capital social en las pymes, por tanto, el estudio se orienta desde el fenómeno mismo en el cual está circunscrito y su influencia directa en el objeto de estudio.

Desde esta perspectiva, de Briones (2007), se concibe una investigación de enfoque pospositivista, porque se estructura a partir de los sucesivos hallazgos que se van realizando durante el transcurso de la investigación, es decir, sobre la marcha de ésta. La validación de las conclusiones obtenidas se hace a través del diálogo, la interacción y la vivencia, las que se van concretando mediante consensos nacidos del ejercicio sostenido de los procesos de observación, reflexión, diálogo, construcción de sentido compartido y sistematización.

Una vez analizado el enfoque pospositivista de la investigación, es importante 
considerar los planteamientos de Guba y Lincoln citado por Escobedo (2005), quienes explican los paradigmas como aquellos vistos como un conjunto de creencias básicas (o metafísicas) relacionadas con los principios últimos. Representan una visión del mundo que define, para quien los detenta, la naturaleza del "mundo", el lugar de los individuos en él, y el rango de posibles relaciones con ese mundo y sus partes, tal como lo hacen las cosmologías y las teologías. Las creencias son básicas en el sentido de ser aceptadas sobre la base de la fe.

Según lo anterior, para hablar de paradigma epistemológico de una investigación, se debe tratar de entender como el hombre, en su necesidad de obtener conocimiento, se ocupa de los problemas históricos, psicológico, sociales y demás que tengan injerencia en su ser, en especial, como considera la objetividad, la verdad o la realidad para lograr la episteme de las situaciones analizadas.

Lo anterior puede interpretarse, tomando en cuenta que el paradigma epistemológico de la investigación vendría a ser el cualitativo, cuya estructura coherente constituida por una red de conceptos a través de los cuales ven su campo los científicos, compuesta por creencias metodológicas y teóricas entrelazadas que permiten la selección, evaluación, crítica de temas, problemas o métodos. La misma, involucra el compromiso entre los miembros de una comunidad científica, todo lo cual implica una definición específica del campo de la ciencia correspondiente, se expresa en una tradición orgánica de investigación. Por todo lo expresado, la corriente pospositivista se enmarca en el concepto de paradigma cualitativo y dentro de esta orientación epistémica existe el enfoque fenomenológico hermenéutico el cual se puede apreciar como un paradigma, en la medida que su origen obedece a hechos históricos, los cuales condujeron a los pensadores, académicos e investigadores de la época a generar una perspectiva diferente para resolver los problemas de la sociedad, dejando las bases para abordar el análisis de dicha situaciones desde técnicas diferentes, hoy constituyen metodologías apropiadas para estudiar el objeto desde la realidad misma en el cual está subscrito.

Si se considera desde su origen, León (2009) explica la fenomenología como una 
reflexión filosófica la cual se fundamenta firmemente la objetividad del saber mediante un método, cuya principal regla es dejar que las cosas mismas se hagan patentes en su contenido esencial, a través de una mirada intuitiva, tal como se dan inmediatamente para el que las vive y poniendo entre paréntesis el juicio sobre la validez de los presupuestos, opiniones o interpretaciones acerca de ellas.

En tal sentido, la fenomenología permite establecer los cimientos para el análisis y la construcción del conocimiento, partiendo desde la comprensión de la situación en la cual el objeto de estudio se desarrolla, para posteriormente emitir consideraciones a partir de la percepción del sujeto investigador, el cual está presente pero no se involucra, haciendo abstracción del fenómeno que le permita mediante juicio crítico entender la realidad.

Si lo se examina desde la postura hermenéutica, este enfoque enriquece cualquier análisis, puesto que, considera factores del objeto los cuales no eran tenidos en cuenta por los positivistas, es decir, la visión centrada en la realidad del objeto conduce a concentrarse en factores subjetivos, estos interpretados apropiadamente, extraen elementos de análisis, propios de los sujetos objetos de estudios o del fenómeno, esclareciendo aún más la búsqueda del conocimiento que se desea obtener.

Visto de esta forma, el paradigma fenomenológico hermenéutico surge como una composición de dos paradigmas que contribuyen a generar un modelo de pensamientos orientado a descubrir repuestas y soluciones a problemas de investigación, basados en el análisis detallado del objeto de estudio sin intervenir, interpretando el fenómeno en su desarrollo natural.

De acuerdo con Martínez (2009), el enfoque cualitativo presenta su forma propia de entender la muestra de la población estudiada que ofreció la información necesaria para realizar la investigación. La opción ontológica de quienes asumen esta postura exige una muestra que no podrán ser elementos aleatorios, escogidos al azar y descontextualizados, si no por un todo sistémico con vida propia, como es una persona, una institución o grupo social. Por ello se impone la muestra intencional, donde se prioriza la profundidad sobre la extensión y la muestra se reduce a su amplitud numérica. 
De acuerdo a lo anterior, en la investigación cualitativa la muestra puede ser variada, pero su escogencia depende de su relación con el objeto de estudio, por tanto, no interesa escoger un tamaño representativo en cantidad de la población, si no por el contrario, tomar unidades pequeñas que permitan entender holísticamente la realidad, en la medida, que se consideran valores subjetivos para este tipo de investigación los cuales resultan cruciales para lograr un conocimiento lo más acertado posible del fenómeno.

De esta manera los informantes se eligieron teniendo en cuenta la pertinencia de trabajar con directivos en PYMES que poseen el mismo nivel académico, con supuestos conocimientos y manejo de temas gerenciales, en particular parte del nivel administrativo alto de la organización, lo cual para este estudio se considera nivel estratégico, se toma este criterio de forma deliberada por la temática de estudio, sujetos con experiencia en talento humano, vinculación en el medio de estudio e imparcialidad por su grado de formación.

De este modo, la selección de los informantes claves los efectos de la presente investigación, fueron tomados de una población de cuatro empresas de pequeña y mediano tamaño en Colombia, estos se escogen sin ningún criterio muestral y estuvo representado por un grupo de personas que desempeñan funciones gerenciales.

Es necesario tomar en cuenta la manera en que el investigador se apropia de la información para poder analizarla, en este sentido, Tamayo (2012), define la técnica como un conjunto de procedimiento para el aprovechamiento de los elementos que rodean los fenómenos sobre los cuales se investiga, También, considera que es un grupo de mecanismos, medios o sistema de dirigir, recolectar, conservar, reelaborar, transmitir datos.

En este sentido, coherente con la metodología expresada previamente, se considera que las técnicas más apropiadas para recolectar información del objeto de estudio son la observación participante y la entrevista semiestructurada, en la medida que consideran factores subjetivos como elementos fundamentales para lograr el propósito de la investigación, sin pasar por alto aquellos componentes considerados objetivos, obteniendo una mayor objetividad en el análisis.

Para Dewalt y Dewalt (2007), la observación participante es el proceso que faculta 
a los investigadores a aprender acerca de las actividades, así como personas en estudio en el escenario natural a través de la observación y participando en sus actividades, provee el contexto para desarrollar directrices de muestreo, así como guías de entrevistas. En cuanto a la entrevista semiestructurada, Mayan (2010), considera que es una técnica la cual permite recolectar datos de los individuos participantes a través de un conjunto de preguntas abiertas formuladas en un orden específico. En contraste con la entrevista no estructurada, la entrevista semiestructurada se enfoca sobre una serie de preguntas las cuales el investigador hace a cada participante.

\section{CONSIDERACIONES FINALES}

El desconocimiento de la naturaleza de los mercados, las dificultades para anticiparse a las dificultades y la lenta reacción ante la búsqueda de soluciones, así como el poco aprovechamiento de las oportunidades, han sido por mucho tiempo características recurrentes de las PYMES. Esto, aunado a las tendencias dinámicas, por lo que, hacen necesario la adopción de un nuevo e innovador modo de producir, así como la utilización de nuevas herramientas de trabajo, creación de nuevas estructuras organizacionales y el desarrollo de nuevas conductas laborales y empresariales.

Sin embargo, las PYMES están obligadas actualmente a actuar de forma conjunta o asociativa para poder generar y participar de los nuevos modelos económicos que les den mayor volumen de producción y comercialización; con la visión de insertarse en mercador específicos, Para esto, deben ser partícipes con principios y acciones firmes, que le den garantía de éxito en la economía del siglo XXI, en consecuencia, es importante que la gestión estratégica como plataforma impulsora del capital social en las pymes tenga en cuenta que se está ante una realidad en la cual la competencia está cada vez más presente, tanto a nivel local como global, por lo que resulta imprescindible prestar especial atención a los más factores que inciden en el mejoramiento o sostenibilidad de la competitividad.

Es necesaria entonces, una transformación radical en las estrategias y modalidades empresariales, ya que estos cambios son necesarios no sólo en los aspectos internos de la empresa, sino que también aspectos relacionados con los 
agentes externos, políticas gubernamentales, entorno en que se desarrolla la actividad empresarial y el medio ambiente; razón por la cual la gestión estratégica de las empresas debe mantenerse capacitado para poder ser impulsor del capital social de la nueva generación de pymes.

Aunado a lo anterior, La PYME, dentro de sus características de gran flexibilidad ante los continuos cambios (externos e internos), la baja inversión por puestos de trabajo productivo, además de la intensidad en la utilización de mano de obra, está llamada a ser una de los principales generadores de valor agregado, así como a dinamizar la economía del futuro.

Otro aspecto concluyente es los obstáculos para acceder al financiamiento de las PYME, ya que estas se encuentran con altos costos de créditos, falta de confianza de los bancos respecto de los emprendimientos (proyectos), la burocracia de los intermediarios financieros, además de la petición de excesivas garantías, por lo que, se puede afirmar que el acceso de las PYME al financiamiento es bajo, si se toma en consideración su actual y potencial capacidad inversora, por lo tanto existe una demanda insatisfecha elevada.

\section{REFERENCIAS CONSULTADAS}

1. Briones, G. (2007). Métodos y técnicas de investigación para las Ciencias Sociales. México D.F. Trillas.

2. Chiavenato, I. (2009). Comportamiento Organizacional. México D. F.: McGraw Hill.

3. Cleri (2013). El Libro de las Pymes. Ediciones Granica S.A. Naucalpan, México.

4. Corma, F. (2012). Innovación, innovadores y empresa innovadora. Editorial Diaz de Santos. Buenos Aires, Argentina.

5. Cortina y Martínez (2008). Construir confianza: ética de la empresa en la sociedad de la información y comunicación. Editorial Trotta, S.A. Madrid, España.

6. Dewalt, K., y Dewalt, B. (2007). Participant observation: a guide for fieldworkers. Walnut Creek, CA: Alta Mira Press. Editorial College. México.

7. Escobedo, J. (2005). Paradigma Epistemológico e Inferencias Lógicas en la 
Investigación Demográficas. XXV Conferencia Internacional de Población. Tours.

8. Etkin, J. (2014). Capital social y valores en la organización sustentable. Ediciones Granica. Naucalpan, México.

9. Francés (2008). Capital Social y organizaciones no lucrativas. Editorial AHS. México.

10.George y Jones (2010). El capital social campesino en la gestión del desarrollo rural. Editorial CEPAL. Santiago de Chile, Chile.

11. Lechner (2010). Obras escogidas: Crisis del Estado en America Latina. LOM Ediciones. Primera edición. Santiago de Chile, Chile.

12. León, E. (2009). El giro hermenéutico de la fenomenológica en Martín Heidegger. Polis.

13. Martínez. (2009). Ciencia y Arte en la Metodología Cualitativa. México D.F.: Editorial Trillas.

14. Mayan, M. (2010). Una Introducción a los Métodos Cualitativos: Módulo de Entrenamiento para Estudiantes y Profesores. Iztapalapa, México: Qual Institute Press.

15. Olcese, Rodríguez y Alfaro (2008). Manual de la empresa responsable y sostenible. Conceptos, ejemplos y herramientas de la RSC o de la Empresa. Madrid, McGraw Hill.

16. Portes, A. (2009). Sociología económica, una investigación sistemática. Primera Edición, Centro de Investigaciones sociológicas. Montalbán, Madrid, España.

17. Putnam, R. (2007). Capital social, trabajo y juventud. Editorial Complejo Editores. Buenos Aires, Argentina.

18. Robbins, S. (2008). Fundamentos de comportamiento organizacional. Quinta Edición. México.

19. Sallenave, JP (2010). Gerencia y planeación estratégica. Grupo Editorial Norma. Colombia.

20. Sandin, M. (2009). Investigación cualitativa en educación: fundamentos y tradiciones. Editorial McGraw-Hill. México.

21. Strickland (2009). Desarrollo Profesional de Instrucción Diferenciación: Una Herramienta Acción ASCD. 
22. Tamayo, M. (2012). Diccionario de la Investigación Científica. México: Limusa

23. Thompson, Strickland y Gamble (2010). Crafting and Executing Strategy: The Quest for Competitive Advantage: Concepts and Cases. Traducción: La elaboración y ejecución de la estrategia: La búsqueda de la ventaja competitiva: Conceptos y Casos.

24. Villafañe, J. (2005). Imagen positiva: gestión estratégica de la imagen de las empresas. Editorial Pirámide. Madrid, España.

\section{REFERENCES CONSULTED}

1. Briones, G. (2007). Methods and techniques for social science research. Mexico DF. Threshing.

2. Chiavenato, I. (2009). Organizational behavior. Mexico D. F .: McGraw Hill.

3. Cleri (2013). The Book of Pymes. Ediciones Granica S.A. Naucalpan, Mexico

4. Corma, F. (2012). Innovation, innovators and innovative company. Editorial Diaz de Santos. Buenos Aires, Argentina.

5. Cortina and Martínez (2008). Build trust: ethics of the company in the information and communication society. Editorial Trotta, S.A. Madrid Spain.

6. Dewalt, K., and Dewalt, B. (2007). Participant observation: a guide for fieldworkers. Walnut Creek, CA: Alta Mira Press. Editorial College. Mexico.

7. Escobedo, J. (2005). Epistemological Paradigm and Logical Inferences in Demographic Research. XXV International Population Conference. Tours

8. Etkin, J. (2014). Social capital and values in the sustainable organization. Granica editions. Naucalpan, Mexico.

9. French (2008). Social Capital and non-profit organizations. Editorial AHS. Mexico.

10. George and Jones (2010). Peasant social capital in the management of rural development. Editorial CEPAL. Santiago de Chile, Chile.

11. Lechner (2010). Selected works: Crisis of the State in Latin America. LOM Editions. First edition. Santiago de Chile, Chile. 
12. León, E. (2009). The hermeneutic turn of the phenomenological in Martin Heidegger. Polis.

13. Martínez. (2009). Science and art in the qualitative methodology. Mexico D.F .: Editorial Trillas.

14. Mayan, M. (2010). An Introduction to Qualitative Methods: Training Module for Students and Teachers. Iztapalapa, Mexico: Qual Institute Press.

15. Olcese, Rodríguez and Alfaro (2008). Manual of the responsible and sustainable company. Concepts, examples and tools of the CSR or the Company. Madrid, McGraw Hill.

16.Portes, A. (2009). Economic sociology, a systematic investigation. First Edition, Center for Sociological Research. Montalbán, Madrid, Spain.

17. Putnam, R. (2007). Social capital, work and youth. Publishers Complex Editorial. Buenos Aires, Argentina.

18. Robbins, S. (2008). Fundamentals of organizational behavior. Fifth edition. Mexico.

19.Sallenave, JP (2010). Management and strategic planning. Norma Editorial Group. Colombia.

20.Sandin, M. (2009). Qualitative research in education: fundamentals and traditions. McGraw-Hill. Mexico.

21.Strickland (2009). Professional Development of Instruction Differentiation: An ASCD Action Tool.

22. Tamayo, M. (2012). Dictionary of scientific investigation. Mexico: Limusa.

23. Thompson, Strickland and Gamble (2010). Crafting and Executing Strategy: The Quest for Competitive Advantage: Concepts and Cases. Translation: The elaboration and execution of the strategy: The search for competitive advantage: Concepts and Cases.

24. Villafañe, J. (2005). Positive image: strategic management of the image of companies. Editorial Pyramid. Madrid Spain. 